\title{
Free Space and Speed Humps Detection using Lidar and Vision for Urban Autonomous Navigation
}

\author{
C. Fernández, M. Gavilán, D. F. Llorca, I. Parra, R. Quintero, A. G. Lorente, Lj. Vlacic, M. A. Sotelo
}

\begin{abstract}
In this paper, a real-time free space detection system is presented using a medium-cost lidar sensor and a low cost camera. The extrinsic relationship between both sensors is obtained after an off-line calibration process. The lidar provides measurements corresponding to 4 horizontal layers with a vertical resolution of 3.2 degrees. These measurements are integrated in time according to the relative motion of the vehicle between consecutive laser scans. A special case is considered here for Spanish speed humps, since these are usually detected as an obstacle. In Spain, speed humps are directly related with raised zebra-crossings so they should have painted white stripes on them. Accordingly the conditions required to detect a speed hump are: detect a slope shape on the road and detect a zebra crossing at the same time. The first condition is evaluated using lidar sensor and the second one using the camera.
\end{abstract}

\section{INTRODUCTION}

According to the Spanish road safety annual report [1] the number of accidents on road and urban areas has been decreased by $52 \%$ and $36 \%$ respectively, in the last 6 years. The report demonstrates that the total number of casualties in Spain in 2009 was 127.680 and $49 \%$ of accidents occurs in urban areas. Another report of the Spanish government [2] highlights that the human factor is present in most of the accidents: inadequate speed, alcohol, distraction or traffic offence by the driver. To eliminate the human factor and reduce the number of victims, there are companies and research groups working on autonomous vehicles development. Some of these autonomous vehicles have participated in international competitions in urban scenarios [3].

Perception of the environment is one of the most important tasks in autonomous navigation because it is an input to many modules of the vehicle, such as the path planning module. Following previous publications about free space [4] [5] [6], in this paper, a specific module is presented to detect free space in front of the vehicle using a lidar sensor for urban scenarios.

As mentioned above, inadequate speed is one of the most repeated factors in urban accidents in Spain. For this reason, public administrations have installed plenty of speed humps to reduce the vehicle speed and, as a consequence, the number of accidents. A speed hump is a rounded device used to reduce vehicle speed. Speed humps are placed across the road to slow down traffic and they are often installed

C. Fernández, M. Gavilán, D. F. Llorca, R. Quintero, I. Parra, A. G. Lorente and M. A. Sotelo are with the Computer Engineering Department, Polytechnic School, University of Alcalá, Madrid, Spain. email: carlos.fernandez, miguel.gavilan, llorca, sotelodaut.uah.es. Ljubo Vlacic is Professor and Director of Intelligent Control Systems Laboratory at Griffith University, Brisbane, Australia. email: l.vlacic@griffith.edu.au in a series of several humps in order to prevent cars from speeding before and after the hump. Generally, speed humps are from 4 to 9 meters in length and span the width of the road. The humps height ranges from 6 to $30 \mathrm{~cm}$.

The massive number of speed humps in Spanish cities makes necessary a specific detection module for this purpose. The vehicle needs to reduce the speed to avoid car damages and pass over the speed hump safely. In this paper, the longitudinal shape of the road is analized to detect obstacles with a similar shape of a speed hump. As depicted in Fig. 1 , the shape of a transversal curb and a speed hump can be very similar, so it is necessary a complementary module to remove detection mistakes.

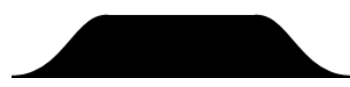

(a) Speed Hump

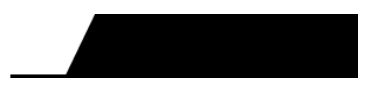

(b) Transversal Curb
Fig. 1. Longitudinal shape of speed hump and transversal curb

In Spain, the norm FOM/3053/2008 [7] specifies the characteristics of speed humps which are directly related with raised zebra-crossings. All of them have to be marked with white painted stripes in order to warn the driver (see Fig. 2). Taking into account this feature, a vision module is developed in this paper to detect the white stripes of speed humps.

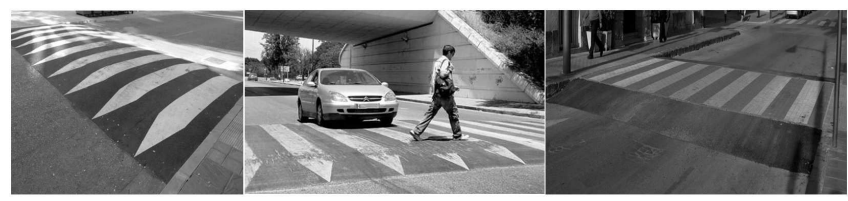

Fig. 2. Some examples of typical raised zebra-crossing of Spanish roads

From the point of view of an autonomous navigation system, raised zebra-crossings can be detected as an obstacle in front of the vehicle (see Fig. 3). The most reasonable manoeuvre in this case is to stop the vehicle. However the vehicle should pass over them to continue the route.

Curbs are delimiters of free space that are usually not detected by standard obstacle detection. A specific method is described in [8] using a stereo pair of cameras and modeling curbs with a cubic spline. In [9] and [10] a general modeling of the visible road surface is presented estimating the road surface using B-splines. A lidar sensor for curb detection is described in [11]. The algorithm is based on the histogram of all the lidar measurements and the maximum peak of 


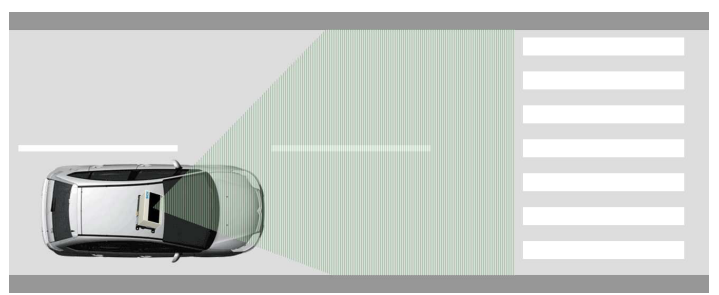

(a) Raised zebra-crossing results in a bad free space estimation

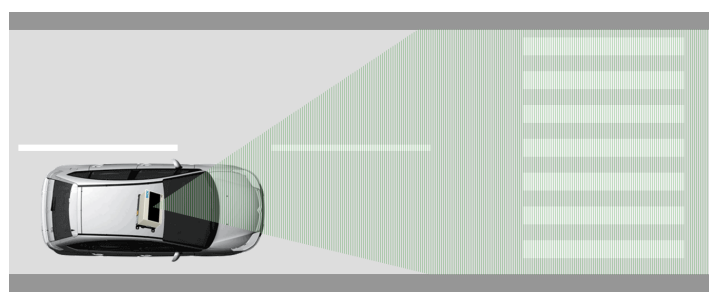

(b) Proposed approach to consider the raised area as free space

Fig. 3. Free space system behaviors

the histogram corresponds with the presence of a curb. The candidate selection method presented in [12] is based on filtering the input signal of the lidar with a differential filter and extracting local maximal and minimal values. For transversal obstacles on the road, the Stanford Team [13] in the Urban Darpa Challenge 2007 [3] installed a lidar sensor on the top of the car rotated 90 degrees to estimate the profile of the road ahead.

There are different detection methods for zebra crossing detection depending on the system hardware. If the system is composed by a stereo pair of cameras, one of the most used technique is the Inverse Perspective Mapping (IPM) [14]. This techique produces a new image that represents the same scene from a different view, commonly a bird-eye view. In the resulting image the parallel stripes of the zebra crossing are easily detected. Considering monocular approaches, [15] uses bipolarity to determine the distribution of white and black pixels in a region of the image and segment the zebra crossing. Furthermore other techniques are used in [16] for zebra crossing segmentation, such as histogram equalization, edge extraction and Fourier Transformation.

\section{SYSTEM DESCRIPTION}

\section{A. Sensors Description}

Autonomous vehicles are usually equiped with three types of sensors for surrounding reconstruction: vision, lidar and radar. The common radar sensors applications are for long distance obstacle detection in highways. The application presented in this paper is related with urban scenarios, so radar sensors are not used on it. Vision provides rich scene information of colours and shapes but the performance of a single camera for distance estimation is not good enough. For this reason, zebra crossings are detected using a single camera and lidar is used for accurate free space computation. The selected camera is a Point Grey Firefly MV OEM BoardLevel and it provides $640 \times 480$ images at $30 \mathrm{fps}$.
The laser is a Sick LD-MRS HD with a scanning range of 30 meters which is enough to drive in urban environments at low speed. The frequency of $50 \mathrm{~Hz}$ with a 0.5 degrees resolution is good for safety obstacle detection and it can work under extreme weather conditions (heavy rain, dust and snow). It has 4 layers with a vertical angle of 3.2 degrees for obstacle height estimation and a better surrounding reconstruction.

\section{B. Lidar and Camera Calibration}

In order to know the projection of the lidar measurements in the camera images it is necessary to know the extrinsic relationship between both sensors. To obtain the extrinsic relationship, we place a planar chessboard pattern in front of the vehicle which will be visible to both the camera and the lidar. Fig. 4 depicts a general view of calibration setup. Laser points are manually identified onto the chessboard pattern by using a labelling tool.

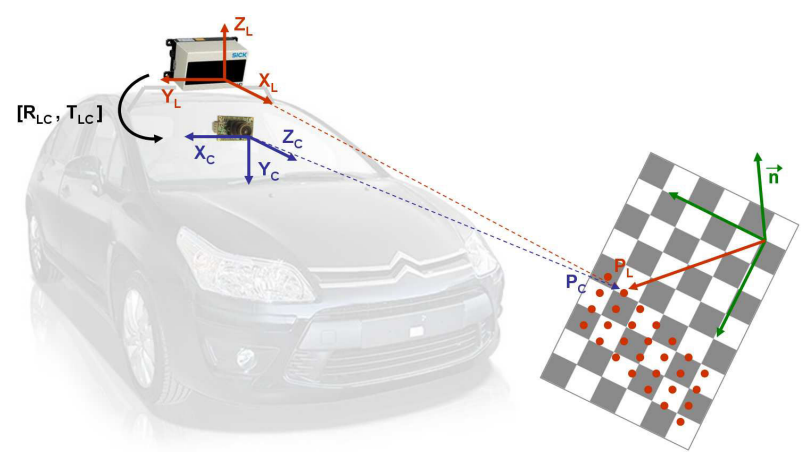

Fig. 4. Calibration setup: a planar chessboard pattern is located in front of the vehicle. Different poses are obtained. The goal is to find the rotation $R_{L C}$ and the translation $T_{L C}$ which transforms points from the lidar reference to the camera coordinate system.

The calibration process needs extrinsic plane to camera parameters of different poses of the pattern, as well as the laser points on the chessboard, which are only a portion of the whole laser measurements. For each pose, in the camera reference system, the calibration plane can be defined by the normal vector $\vec{n}_{i}$, given by the 3 rd column of rotation matrix $R_{3 i}=\left[r_{13 i}, r_{23 i}, r_{33 i}\right]$, and a $3 \mathrm{D}$ point, that is given by the translation vector $T_{i}$ :

$$
\vec{n}_{i} \cdot\left(P_{C i j}-T_{i}\right)=0
$$

where $P_{C i j}$ represents any $3 \mathrm{D}$ point of the plane w.r.t. the camera for the $\mathrm{i}^{\text {th }}$ pose. Extrinsic parameters between the plane and the camera are obtained after monocular camera calibration. Given $P_{C i j}$ in the camera coordinate system, we can determine its coordinate in the lidar reference $P_{L i j}$ by:

$$
P_{C i j}=R_{L C} P_{L i j}+T_{L C}
$$

where $R_{L C}$ and $T_{L C}$ define the relative position and orientation between of the lidar w.r.t. the camera coordinate system. These are the extrinsic parameters we want to estimate. By using expression 2 in equation 1 we have: 


$$
\vec{n}_{i} \cdot\left(R_{L C} P_{L i j}+T_{L C}-T_{i}\right)=0
$$

For each one of the poses we manually select the laser points that lie on the plane. Using all the points corresponding to the $\mathrm{N}$ poses we obtain an overconstrained $A x=b$ system with several linear equations in the unknown parameters $\left[R_{L C}, T_{L C}\right]$. Since the computed matrix $R_{L C}$ may not meet the properties of a rotation matrix, we obtain the nearest orthogonal matrix $\hat{R}_{L C}$ by minimizing Frobenius norm of the difference $\hat{R}_{L C}-R_{L C}$, subject to $\hat{R}_{L C} \hat{R}_{L C}{ }^{T}=I$.

As suggested in [17], the above solution is obtained by minimizing an algebraic distance which is not directly related to the measurement. Accordingly, this solution is used as the initial guess of a nonlinear minimization on the Euclidean distances from laser points to the checkboard planes, which is more physically meaningful. The initial guess can be also obtained by means of SVD, which provides a faster but less accurate solution (SVD can be used if real time requirements would be needed). Given the different poses of the chessboard pattern, an error function $f\left(R_{L C}, T_{L C}\right)$ is defined as the sum of the distances of every laser point $j$ located on the plane of pose $i$ :

$$
\sum_{i} \sum_{j}\left|n_{i} \cdot\left(R_{L C} P_{L i j}+T_{L C}-T_{i}\right)\right|^{2}
$$

As described in [17], equation 4 is minimized by using the well-known Levenberg-Marquardt optimization method.

\section{Free Space Detection}

In an autonomous navigation system it is necessary to know the free space in front of the vehicle for path planning estimation. In this paper a lidar-based method is proposed for free space detection using 4 layers at different distances using parallel computing.

We asumme that the road is the lowest smooth surface and the curbs limit the road. Reading laser measurements sequentially, strong changes on road surface correspond with curb locations or bigger obstacles. In order to find those strong changes in the road surface, the angle between three points is computed. Given 3 points of laser measurements $P_{i}=\left(X_{i}, Y_{i}, Z_{i}\right)$ and vectors $\vec{u}_{i}, \vec{v}_{i}$, the angle formed by them is defined as dot product of those vectors.

$$
\begin{gathered}
\overrightarrow{u_{i}}=\overrightarrow{P_{i-1} P_{i}} \quad, \quad \overrightarrow{v_{i}}=\overrightarrow{P_{i} P_{i+1}} \\
\theta_{i}=\arccos \left(\frac{\overrightarrow{u_{i}} \cdot \overrightarrow{v_{i}}}{\left\|\overrightarrow{u_{i}}\right\| \cdot\left\|\overrightarrow{v_{i}}\right\|}\right) \\
\Theta_{i}=\sum_{j} \theta_{j} \forall j \in[i-1, i+1]
\end{gathered}
$$

Given a set of curb candidate points $C$, one point $P_{i}$ is considered as a curb candidate when satisfies equation 8 .

$$
P_{i} \in C \Leftrightarrow \Theta_{i}>\gamma
$$

where $\gamma$ is a threshold for the maximum angle of road surface. The curb candidates situated on the smooth surface limits are confirmed as the limits of the road. In Fig. 5 a single layer measurement and camera frame are shown to understand the scene and the profile of the measurements.

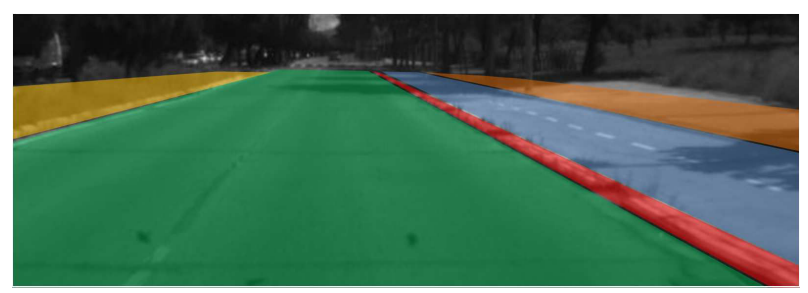

(a) The scene is composed (from left to right) by trees, 6.5 meters wide road, curb, bicycle way, small curb and sidewalk

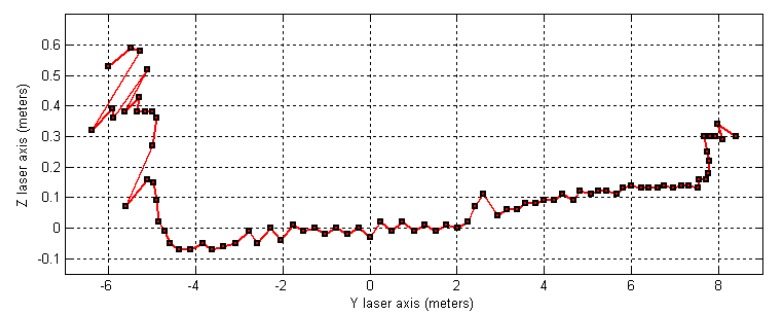

(b) Road profile

Fig. 5. Reading laser measurements sequentially, strong changes on road surface correspond with curb locations or bigger obstacles.

\section{Lidar Measurements Integration}

The lidar provides measurements as far as 15 meters from the front part of the vehicle. These measurements must be updated according to the relative motion of the vehicle between two consecutive laser scans, so that the road limits and the obstacles at closer locations are also considered, achieving a more extensive description of the free space in front of the vehicle. Unlike some recent works, such as [18], where costly IMU devices are employed, the vehicle motion is computed using the data supplied by the CAN bus, namely the yaw-rate and the speed of the vehicle, allowing to maintain a low-cost for the proposed system.

The laser fixed on the vehicle traces a circular motion around a point, known as Instantaneous Center of Rotation (ICR). The existence of this point is ensured by the Ackermann principle [19]. The sensor is not located above the rear wheel axis of the vehicle so it is necessary to take into account the displacement of the sensor from the axis, $d_{L}$. Consequently, besides the transformation between two consecutive vehicle positions, a second transformation between the laser and the vehicle must be considered in the parametrization of the laser motion (see Fig. 6). A further explanation regarding with the motion model as well as a validation of the circular motion constrain in real situations are provided in [20]. Assuming perfect circular motion with rotation angle $\theta$, the direction of the translation is $\phi=\theta / 2$. Following these considerations, the transformation between two consecutive laser positions, $\left[R_{L L^{\prime}}, T_{L L^{\prime}}\right]$, can be obtained as follows: 


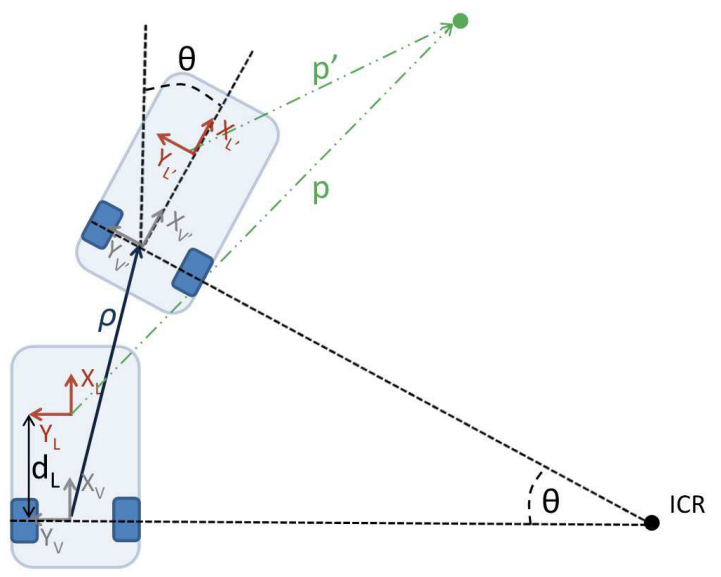

Fig. 6. Relation between laser axes in circular motion.

$$
\begin{aligned}
R_{L L^{\prime}} & =R_{V V^{\prime}}=\left[\begin{array}{ccc}
\cos (\theta) & -\sin (\theta) & 0 \\
\sin (\theta) & \cos (\theta) & 0 \\
0 & 0 & 0
\end{array}\right] \\
T_{L L^{\prime}} & =T_{L V}+T_{V V^{\prime}}+T_{V^{\prime} C^{\prime}} \\
& =\left[\begin{array}{c}
-L \\
0 \\
0
\end{array}\right]+\rho \cdot\left[\begin{array}{c}
\cos (\theta / 2) \\
\sin (\theta / 2) \\
0
\end{array}\right]+d_{L} \cdot\left[\begin{array}{c}
\cos (\theta) \\
\sin (\theta) \\
0
\end{array}\right] \\
= & {\left[\begin{array}{c}
-d_{L}+r h o * \cos (\theta)+d_{L} * \cos (\theta) \\
r h o * \sin (\theta)-d_{L} * \sin (\theta) \\
0
\end{array}\right] }
\end{aligned}
$$

Then, a point $p$ can be transformed to the new laser reference system by means of Equation 11 .

$$
p^{\prime}=R_{L L^{\prime}}^{-1} \cdot p-T_{L L^{\prime}}
$$

The laser measurements are accumulated applying updating steps at each frame $t$. A set measurements, $M(t)$, is available at each frame with all the measurements remaining inside the analyzed space in front of the vehicle:

$$
\begin{aligned}
& m_{t}(t+1)=R_{L L^{\prime}}^{-1}(t) \cdot m_{t}(t)-T_{L L^{\prime}}(t) \\
& M(t)=\left\{m_{t}(t), m_{t-1}(t), \ldots, m_{t-N}(t)\right\}
\end{aligned}
$$

\section{E. Speed Hump Detection}

A speed hump is a rounded device used to reduce vehicle speed and they usually are from 4 to 9 meters length and from 6 to $30 \mathrm{~cm}$ height. Using Spanish norms, the criterion for diferenciating whether an obstacle is a speed hump or not is that the raised zebra crossing should have an elevation profile as depicted in Fig. 1 and also it should have painted white stripes on it. In order to estimate road profile all laser layers are analized but only two are depicted in Fig. 7 to simplify the explanation.

As depicted in Fig. 8 the fixed vertical angle between layers allow to estimate road surface. Integrating measurements

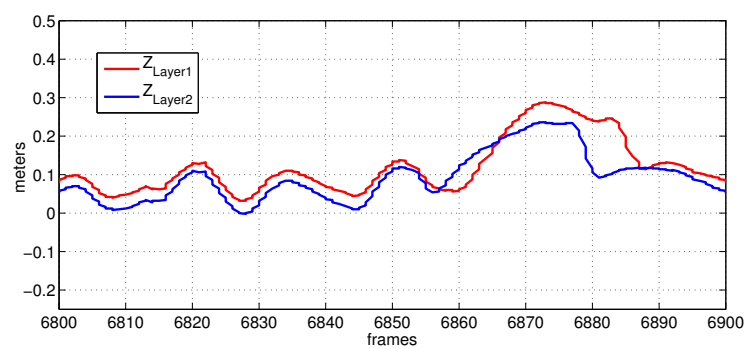

Fig. 7. Measurements pattern detected for speed hump detection. In this example, the distance between layers raises from frame 6878 to frame 6886 That interval of frames corresponds with the obstacle in front of the vehicle. Problem of pitching is present in the measurements. In future works it will be fixed installing gyroscopes to compensate car movement.

over time, when the vehicle is aproaching to a speed hump the measurements start raising slowly and few frames later they fall rapidly. Nevertheless when there is not an obstacle on the road, $\mathrm{Z}$ axis difference between layers is very small. This method detects obstacles with a slope shape, therefore when the vehicle is entering in a roundabout the profile shape of the roundabout is similar to a speed hump. For this reason, a complementary module is needed to avoid false positive detections.

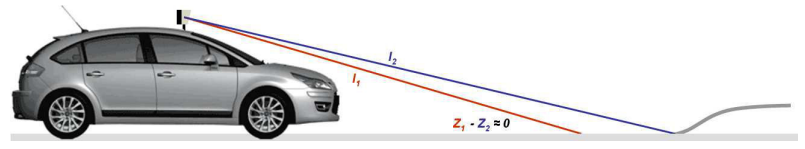

(a) When the vehicle is driving in a road without speed humps, the difference between layers in $\mathrm{Z}$ axis is very small.

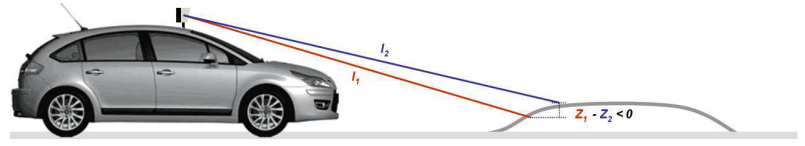

(b) When the vehicle is aproaching a speed hump, the difference between layers in $\mathrm{Z}$ axis start raising but it is difficult to diferenciate a speed hump and uphill.

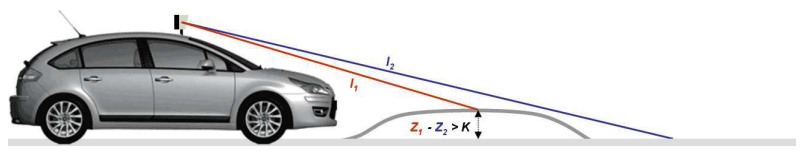

(c) When the laser beam impacts behind speed hump, the difference between layers in $\mathrm{Z}$ axis is large and easy to detect.

Fig. 8. Road surface estimation.

\section{F. Zebra Crossing Detection}

As commented in section II-E, the criteria for diferenciating whether an obstacle is a speed hump or not is that the raised zebra crossing should have an elevation profile and also it should have painted white stripes on it. The first condition is evaluated using the algorithm described in the previous section and the second condition is evaluated using the criteria explained below.

1) Region of interest: Some elements appear in the original camera image that are not relevant for zebra crossing 
detection, such as the sky, buildings and ego-vehicle. Consequently a region of interest is applied to the original image to avoid false positive detections.

2) Edge extraction: The rectangular shape of the white stripes over the dark road allows to apply edge detection algorithms. In this paper, the Canny method is applied to extract edges and the result is a one pixel width edges image, see Fig. 9(a).

3) Contour extraction: The disjointed edges are fixed applying an operation of dilation. After the operation of dilation, contours are detected and filled to get a binary image with stripes candidates in white and background in black, see Fig. 9(b).

4) Image row analysis: 20 rows of the resulted image are analized and 5 of them should be positive to confirm the presence of a zebra crossing, see Fig. 9(c).

A sequence of pulses is obtained analyzing the values of each row. It is considered positive when the number of consecutive positive and negative pulses with the same width is greater than a threshold, see Fig. 9(d).

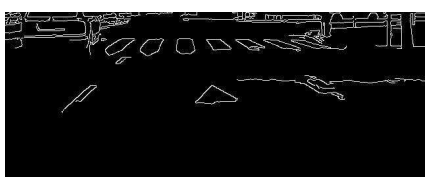

(a) Edge extraction

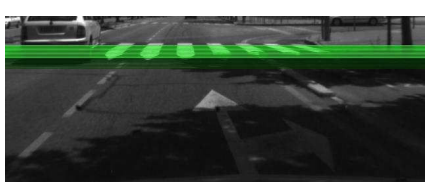

(c) Analized rows

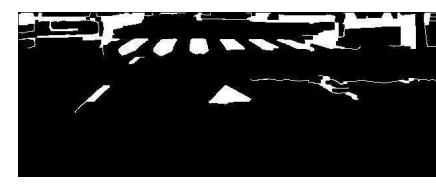

(b) Contour extraction

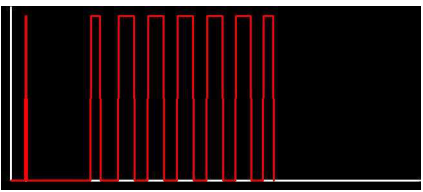

(d) Positive and negative pulses
Fig. 9. Steps of zebra crossing method

\section{RESULTS}

Autonomous vehicles are real-time systems because their actions have to be done into a specific time interval. The real-time restrictions of the module presented in this paper are imposed by the highest frequency of the module, in this case the laser. The laser was been configured to work at $50 \mathrm{~Hz}$, so the maximum processing time has to be less than $20 \mathrm{~ms}$. The processsing times detailed in Table I are obtained using a Intel Quad Core at $2.83 \mathrm{GHz}$ with $3.25 \mathrm{~GB}$ of RAM memory at $667 \mathrm{MHz}$. Most of CPU time (76.15\%) corresponds to image processing for zebra crossing detection. The second most complex task is the geometric transformations to convert from laser coordinate system to ego-vehicle coordinate system and from ego-vehicle coordinate system to image coordinates. Speed humps and free space detection algorithms require a little processing time $(6.12 \%)$, hence the complete module is computed in $6.48 \mathrm{~ms}$. According to processing time, the module frequency is $154 \mathrm{fps}$, so the system satisfies real time constraints.

In Spain, maximum speed is limited to $50 \mathrm{~km} / \mathrm{h}$ in urban areas so the maximum car speed in the analized sequences is
TABLE I

PROCESSING TIME

\begin{tabular}{|l|c|c|}
\hline TASK & CPU TIME (ms) & $\%$ TIME \\
\hline Zebra Crossing Detection & 4.93 & $76.15 \%$ \\
\hline Geometric Transformation & 1.11 & $17.27 \%$ \\
\hline Speed Hump Detection & 0.38 & $5.97 \%$ \\
\hline Free Space Detection & 0.015 & $0.24 \%$ \\
\hline Others & 0.02 & $0.35 \%$ \\
\hline Total & 6.48 & $100.00 \%$ \\
\hline
\end{tabular}

$40 \mathrm{~km} / \mathrm{h}$. Driving at this speed, the system is able to detect $100 \%$ of speed humps 12 meters before the car is driving over them. Detection distance is enough to brake smoothly and pass over the speed hump at $20 \mathrm{~km} / \mathrm{h}$. The system has been tested along $10 \mathrm{~km}$ urban route with the presence of 10 speed humps (raised zebra-crossings), 10 roundabouts and 58 zebra crossings. Some result images are shown in Fig. 10. All speed humps have a zebra crossing painted on them but there are also zebra crossings which are not speed humps. 100\% of speed humps (lidar positive detection + zebra crossing vision detection) and $94 \%$ of zebra crossings are detected (lidar negative detection + zebra crossing vision detection), so the goal of detecting speed humps to reduce the vehicle speed and pass over them safely is reached.

If road width is enough for driving safely, it is shown in green on the top center of the images, otherwise it is shown in red. When the system detects a zebra crossing or speed hump, an alert message is shown in green for driver warning. In Fig. 10(a), there are cars parked on the left and right sides with degradated zebra crossings along the street. All of them are detected and free-space is adapted to parked cars. In Fig. 10(b), the road is limited on the left side with a big curb and some trees. The right side is limited with a small curb near a bike-way, which is correctly detected. In Figs. 10(c) and $10(\mathrm{~d})$, the vehicle is driving in a street with some zebra crossings. In these cases, the zebra crossings are not speed humps, they are just normal zebra crossings, so the vehicle can drive normally. The zebra crossing depicted in Fig. 10(e) is painted on a speed hump. In this situation, the system detects that the obstacle has white stripes on it, therefore the car should pass over it. In Fig. 10(f), a vehicle is overtaking the ego-vehicle and free-space is correctly adapted to the dynamic obstacle. At the same time, the vehicle is aproaching a speed hump and it is correctly detected. In Fig. 10(g), the vehicle is aproaching a roundabout. The obstacle is detected but the zebra crossing is not detected, so the system considers it as a real obstacle instead of a speed hump. Finally, in Fig. 10(h), the car is driving in a street wide enough but a bus does not allow to drive safely. In this case, the estimated road width is less than the minimum space, so the vehicle should stop until the bus allows it to drive safely.

\section{CONCLUSIONS AND FUTURE WORKS}

A free space detection system is presented in this paper using an outdoor laser sensor. The system is focused on speed humps detection and for this task, according to Spanish 


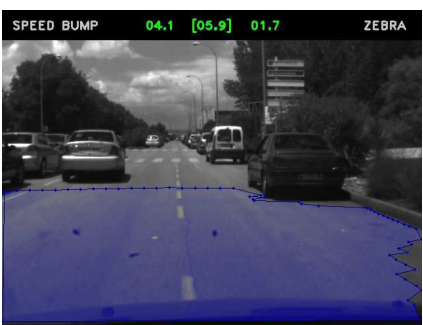

(a)

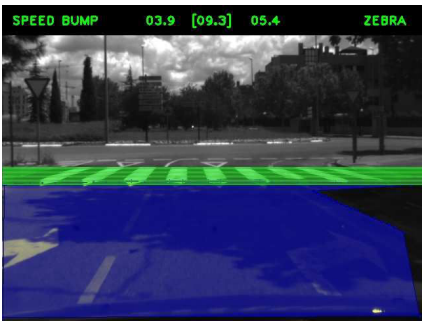

(e)

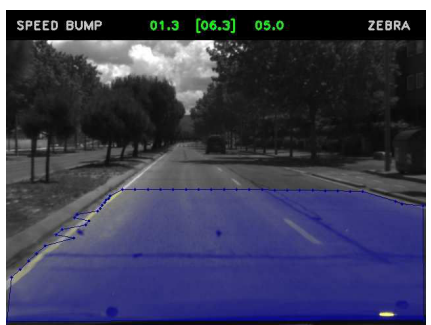

(b)

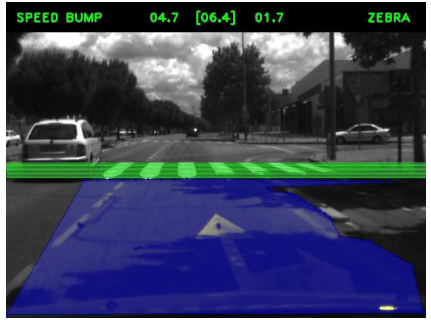

(f)

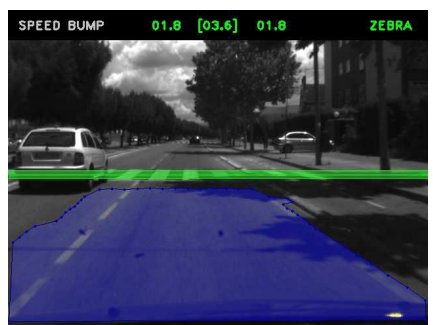

(c)

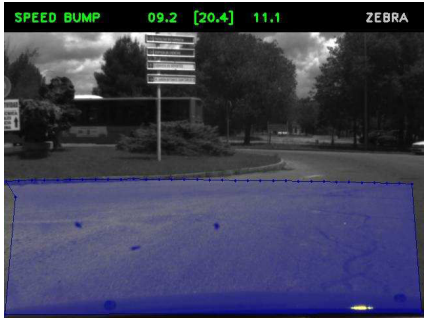

(g)

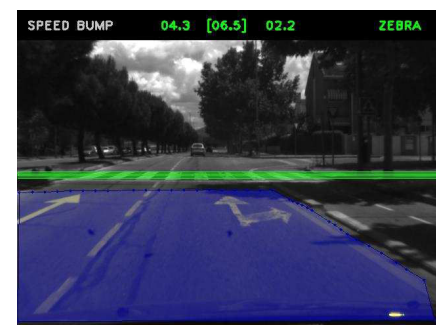

(d)

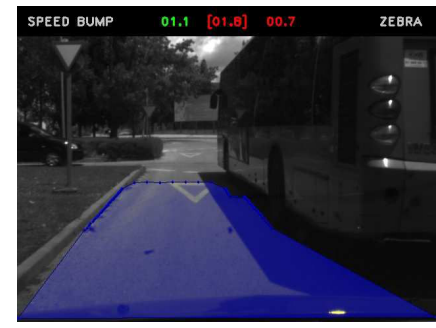

(h)

Fig. 10. Results of analized sequences.

normative, a zebra crossing detection system is required to verify the presence of speed humps. One of the most important constraints is the real-time constraint. This configuration needs to work at $50 \mathrm{fps}$ and the system is able to work at $154 \mathrm{fps}$, so a fast detection system is reached.

For a more reliable perception of the environment, a 3D occupancy grid approach is proposed to eliminate outliers of measurements using probabilistic occupancy estimation. Furthermore zebra crossing detection is related with pedestrian safety therefore an improvement of zebra crossing algorithm is proposed as future work. The accuracy of the system is related with the angular resolution of the laser so different configuration tests are proposed to compare results.

\section{ACKNOWLEDGMENTS}

This work was supported by the Spanish Ministry of Science and Innovation under Research Grant ONDA-FP TRA2011-27712-C02-02.

\section{REFERENCES}

[1] D. G. de Tráfico, "Accidentes de tráfico en zona urbana. españa 2009," Gobierno de España, Ministerio del Interior, Madrid, Spain, Annual Report, 2010.

[2] —-, "Las principales cifras de la siniestralidad vial. españa 2009," Gobierno de España, Ministerio del Interior, Madrid, Spain, Annual Report, 2010.

[3] DARPA. (2007) Defense advanced research projects agency. [Online]. Available: www.darpa.mil

[4] M. A. Sotelo, F. J. Rodriguez, and L. Magdalena, "Virtuous: visionbased road transportation for unmanned operation on urban-like scenarios," Intelligent Transportation Systems, IEEE Transactions on, vol. 5, no. 2, pp. $69-83,2004$.

[5] M. A. Sotelo, F. J. Rodriguez, L. Magdalena, L. M. Bergasa, and L. Boquete, "A color vision-based lane tracking system for autonomous driving on unmarked roads," Auton. Robots, vol. 16, pp. 95-116, 2004.

[6] F. J. Rodríguez, M. Mazo, and M. A. Sotelo, "Automation of an industrial fork lift truck, guided by artificial vision in open environments," Auton. Robots, vol. 5, pp. 215-231, 1998.
[7] BOE, "Instrucción técnica para la instalación de reductores de velocidad y bandas transversales de alerta en carreteras de la red de carreteras del estado," Gobierno de España, Ministerio del Interior, Madrid, Spain, Annual Report 261, 2008.

[8] F. Oniga and S. Nedevschi, "Curb detection for driving assistance systems: A cubic spline-based approach," in Intelligent Vehicles Symposium (IV), 2011 IEEE, 2011, pp. 945 -950.

[9] A. Wedel, H. Badino, C. Rabe, H. Loose, U. Franke, and D. Cremers, "B-spline modeling of road surfaces with an application to free-space estimation," Intelligent Transportation Systems, IEEE Transactions on, vol. 10, no. 4, pp. $572-583,2009$.

[10] C. K. Keller, M. Enzweiler, R. M., D. F. Llorca, C. Schnörr, and D. M. Gavrila, "The benefits of dense stereo for pedestrian detection," Intelligent Transportation Systems, IEEE Transactions on, vol. 12, no. 4, pp. $1096-1106,2011$.

[11] R. Aufrere, C. Mertz, and C. Thorpe, "Multiple sensor fusion for detecting location of curbs, walls, and barriers," in Intelligent Vehicles Symposium, 2003. Proceedings. IEEE, 2003, pp. 126 - 131.

[12] W. Zhang, "Lidar-based road and road-edge detection," in Intelligent Vehicles Symposium (IV), 2010 IEEE, 2010, pp. 845 -848.

[13] M. Montemerlo, J. Becker, S. Bhat, et al., "Junior: The stanford entry in the urban challenge," J. Field Robot., vol. 25, pp. 569-597, 2008.

[14] M. Bertozzi, A. Broggi, P. Medici, P. Porta, and A. Sjogren, "Stereo vision-based start-inhibit for heavy goods vehicles," in Intelligent Vehicles Symposium, 2006 IEEE, 2006, pp. 350 -355.

[15] M. Uddin and T. Shioyama, "Bipolarity and projective invariantbased zebra-crossing detection for the visually impaired," in CVPR Workshops, 2005, p. 22.

[16] S. Sichelschmidt, A. Haselhoff, A. Kummert, M. Roehder, B. Elias, and K. Berns, "Pedestrian crossing detecting as a part of an urban pedestrian safety system," in Intelligent Vehicles Symposium (IV), 2010 IEEE, 2010, pp. $840-844$.

[17] Q. Zhang and R. Pless, "Extrinsic calibration of a camera and laser range finder (improves camera calibration)," in IEEE IROS 2004, vol. 3, 2004, pp. 2301-2306 vol.3.

[18] V. Milanés, J. Naranjo, C. Gonzánez, J. Alonso, and T. de Pedro, "Autonomous vehicle based in cooperative gps and inertial systems," ROBOTICA, vol. 26, pp. 627-633, 2008.

[19] R. Siegwart, I. R. Nourbakhsh, and D. Scaramuzza, Introduction to Autonomous Mobile Robots, 2nd ed. The MIT Press, 2011.

[20] D. Scaramuzza, "1-point-ransac structure from motion for vehiclemounted cameras by exploiting non-holonomic constraints," Int. J. Comput. Vision, vol. 95, pp. 74-85, 2011. 